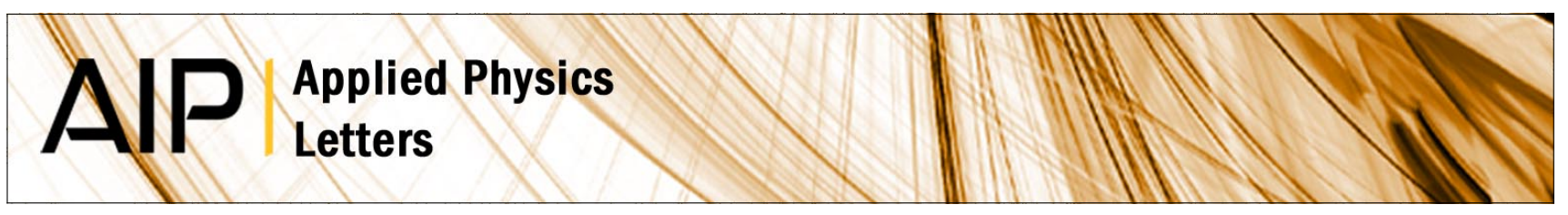

\title{
Enhanced photocurrent in single-walled carbon nanotubes by exciton interactions
}

Satoru Konabe and Susumu Okada

Citation: Appl. Phys. Lett. 102, 113110 (2013); doi: 10.1063/1.4798274

View online: http://dx.doi.org/10.1063/1.4798274

View Table of Contents: http://apl.aip.org/resource/1/APPLAB/v102/i11

Published by the American Institute of Physics.

Additional information on Appl. Phys. Lett.

Journal Homepage: http://apl.aip.org/

Journal Information: http://apl.aip.org/about/about_the_journal

Top downloads: http://apl.aip.org/features/most_downloaded

Information for Authors: http://apl.aip.org/authors

\section{ADVERTISEMENT}

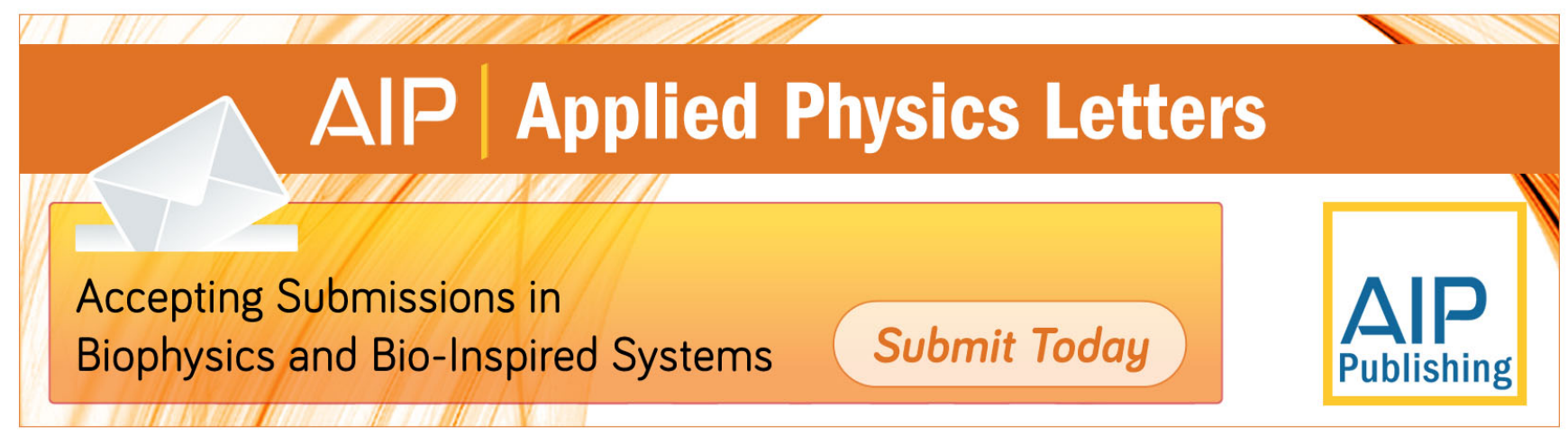




\title{
Enhanced photocurrent in single-walled carbon nanotubes by exciton interactions
}

\author{
Satoru Konabe ${ }^{1,2, a)}$ and Susumu Okada ${ }^{1,2}$ \\ ${ }^{1}$ Graduate School of Pure and Applied Sciences, University of Tsukuba, Tsukuba, Ibaraki 305-8571, Japan \\ ${ }^{2}$ Japan Science and Technology Agency, CREST, 7 Gobancho, Chiyoda, Tokyo 102-0075, Japan
}

(Received 23 October 2012; accepted 11 March 2013; published online 22 March 2013)

\begin{abstract}
We theoretically investigate the photocurrent generation efficiency of single-walled carbon nanotubes by considering the interplay between exciton many-body effects. We calculate the photocurrent by solving rate equations that incorporate the influences of the two competing processes, multiple exciton generation (MEG) and the Auger recombination (AR) processes. We find that MEG substantially enhances photocurrent generation in spite of the competing AR process. Our calculation shows that the generation efficiency is up to $150 \%$ higher than that without MEG. (C) 2013 American Institute of Physics. [http://dx.doi.org/10.1063/1.4798274]
\end{abstract}

The increasing global population and burgeoning economic activity require huge amounts of electrical power. Solar energy is the most promising renewable and sustainable energy source. Although much experimental and theoretical effort has been devoted to developing and designing photovoltaic devices with high power conversion efficiencies, the conversion efficiency of conventional solar cells is fundamentally limited by the Shockley-Queisser (SQ) limit. ${ }^{1}$ Therefore, novel device structures and new materials that can overcome the SQ limit are required to substantially increase the power conversion efficiency of solar cells.

Multiple exciton generation (MEG) is a promising process for exceeding the SQ limit. In low-dimensional materials, a single photon can create an exciton (a bound electron-hole pair) that has a sufficiently high energy that it can generate other excitons by transferring its energy via the strong Coulomb interaction. ${ }^{2-6}$ This contrasts with the process observed in bulk semiconductors for low excitation intensities for which a single photon can create only a single exciton since any excess energy of the photon above the band gap energy is released as heat through exciton-phonon interactions. Carbon nanotubes (CNTs) are ideal materials for realizing MEG since their quasi-one-dimensional structure enhances the Coulomb interaction. ${ }^{7-12}$ Indeed, MEG has recently been experimentally observed in semiconducting single-walled carbon nanotubes (SWCNTs) ${ }^{13-16}$ and has been theoretically studied. ${ }^{17-19}$

Auger recombination (AR) is another important process for photoexcited states of SWCNTs. In AR, an exciton recombines by transferring its energy to another exciton through exciton-exciton scattering. This is one of the most dominant processes in nonradiative relaxation of excitons in SWCNTs under high-intensity photon irradiation. ${ }^{20-25}$ AR simultaneously generates free carriers from strongly bound excitons when scattering excitons have sufficiently high energies to ionize other excitons. ${ }^{26,27}$ In SWCNTs and other low-dimensional materials that have strong Coulomb interaction, the interaction between these two processes results in the highly diverse physics associated with excitons. In

\footnotetext{
${ }^{a)}$ Electronic mail: konabe@comas.frsc.tsukuba.ac.jp
}

particular, photoconductivity in CNTs is determined by competition between these two processes. Although several theoretical studies have investigated photoconductivity in CNTs, ${ }^{26,28-30}$ a unified understanding of photocurrent generation has yet to be attained.

In this letter, we investigate the efficiency of photoelectric conversion in SWCNTs by accounting for possible competition between MEG and AR processes. Our calculations reveal that photocarriers are generated from both single excitons and multiple excitons generated by MEG, despite AR causing recombination of the generated multiple excitons. The results indicate that MEG significantly enhances the photocurrent and is a major pathway for carrier generation in SWCNTs.

Here, we consider the dynamics of carriers and excitons including $\mathrm{MEG}$ and $\mathrm{AR}$ by solving rate equations that describe the exciton and electron (hole) dynamics

$$
\begin{gathered}
\frac{d n_{\mathrm{ex}}}{d t}=G-\frac{n_{\mathrm{ex}}}{\tau_{\mathrm{ex}}}-\Gamma_{\mathrm{A}} L n_{\mathrm{ex}}^{2}-\Gamma_{\mathrm{F}} n_{\mathrm{ex}}, \\
\frac{d n}{d t}=\Gamma_{\mathrm{A}} L n_{\mathrm{ex}}^{2}+\Gamma_{\mathrm{F}} n_{\mathrm{ex}}-\frac{n^{2}}{\tau_{\mathrm{eh}}},
\end{gathered}
$$

where $n_{\mathrm{ex}}$ and $n$ are the exciton and electron (or hole) densities, respectively. Here, we assumed the electron and hole densities are equal to each other. The generation rate $G$ in Eq. (1) is the sum of the single-exciton generation rate and the MEG rate given by Eq. (5). The light polarization is assumed to be parallel to the SWCNT axis. In the present study, we consider only multiple exciton states consisting of two excitons. We phenomenologically include phase relaxation of a single exciton such as induced by the exciton-phonon interaction $^{31}$ as a spectral width of $40 \mathrm{meV}$ in $G$. The radiative lifetimes $\tau_{\mathrm{ex}}$ and $\tau_{\mathrm{eh}}$ are calculated as the spontaneous emission lifetime. $^{32}$ The third and last terms on the right-hand side of Eq. (1) represent the AR process for excitons and exciton dissociation by an external electric field, respectively. Both AR and field dissociation cause free carrier generation in Eq. (2). We solve Eqs. (1) and (2) for the isolated SWCNT with the length of $L=1 \mu \mathrm{m}$ to obtain the carrier density $n$ under 
stationary conditions. The carrier density can be used to calculate the steady photocurrent using

$$
I=2 e \mu F n,
$$

where $F$ is the bias field and the mobility $\mu$ is calculated as in Ref. 33.

Exciton states with an amplitude $Z_{k, q}^{n}$ and an energy $E_{q}^{n}$ are obtained by solving the Bethe-Salpeter equation ${ }^{7,8,34,35}{ }^{q}$

$$
\left(\varepsilon_{k+q}^{c}-\varepsilon_{k}^{v}\right) Z_{k, q}^{n}+\sum_{k^{\prime}} K_{k, k^{\prime}} Z_{k^{\prime}, q}^{n}=E_{q}^{n} Z_{k, q}^{n}
$$

where $K_{k, k^{\prime}}$ is the Coulomb interaction kernel that consists of bare-exchange and screened-direct terms. The quasiparticle energies $\varepsilon_{k}^{c}$ and $\varepsilon_{k}^{v}$ are calculated by applying the randomphase approximation..$^{7,34,35}$ For the Coulomb potential between the $\pi$ orbitals of a SWCNT, we employed the Ohno potential $V(\mathbf{r})=U / \kappa \sqrt{\left(\frac{4 \pi \epsilon_{0}}{e^{2}} U|\mathbf{r}|\right)^{2}+1}$ with $U=11.3 \mathrm{eV}$, which has been used to quantitatively describe the optical responses of SWCNTs. ${ }^{8,34-36}$ The dielectric constant $\kappa=$ 3.3 was used to incorporate screening effects by $\sigma$ electrons and the surrounding environment. All calculations were performed under the tight-binding approximation with nearestneighbor hopping energy of $3.0 \mathrm{eV}$. We consider a $(20,0)$ SWCNT as a representative semiconducting SWCNT.

In the present study, we consider the rate of direct generation of two excitons as the MEG rate in the exciton generation rate $G$ in Eq. (1). Using perturbation theory, the MEG rates are given by ${ }^{19}$

$$
\begin{aligned}
\Gamma_{\mathrm{MEG}}(\omega)= & \frac{2 \pi}{\hbar} \sum_{q}\left|\sum_{n} \frac{\left\langle g\left|\mathcal{H}_{\mathrm{op}}\right| n, 0\right\rangle\langle n, 0|V| 1, q ; 1,-q\rangle}{E_{q}^{1}+E_{-q}^{1}-E_{0}^{n}+i \gamma}\right|^{2} \\
& \times \delta\left(\hbar \omega-E_{q}^{1}-E_{-q}^{1}\right) .
\end{aligned}
$$

We assume that the two excitons in the final state do not interact with each other. The MEG process is described by the following processes (Fig. 1(a)). First, the excited states of a single exciton with zero momentum $|n, 0\rangle$ are generated from the ground state $|g\rangle$ by the exciton-photon interaction, which is denoted by $\mathcal{H}_{\text {op }}$. These excited states then act as intermediate states and resonate with the final states, $|1, q ; 1,-q\rangle$, with a total momentum of zero through the Coulomb interaction $V$.
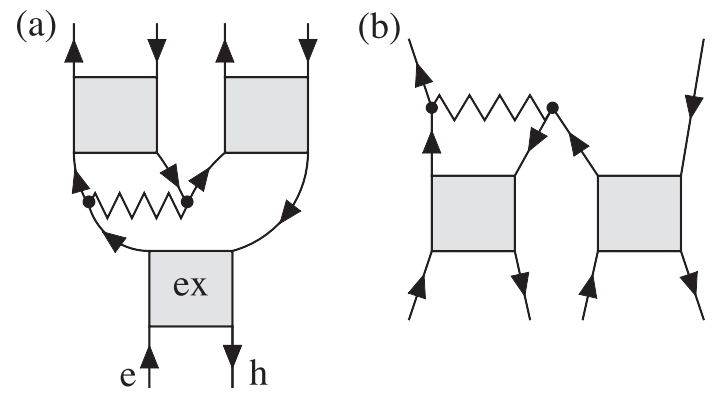

FIG. 1. Exciton scattering processes included in the calculations: Feynman diagrams for (a) MEG and (b) AR. The solid lines indicate the Green's functions of the electron and hole, the wavy lines indicate the Coulomb interaction, and the squares indicate the exciton states.
From Eq. (5), we obtain the threshold energy for MEG occurring at $\hbar \omega=E_{q}^{1}+E_{-q}^{1}$. We phenomenologically consider dephasing processes for the intermediate states by incorporating the dephasing rate of $\gamma=20 \mathrm{meV}$ in the denominator of Eq. (5).

The AR rate for two-exciton scattering is given by ${ }^{20,26}$

$$
\begin{aligned}
\Gamma_{\mathrm{A}}= & \frac{2 \pi}{\hbar} \sum_{q, k}|M|^{2}\left(1-n_{k}^{c}\right)\left(1-n_{k}^{v}\right) \\
& \times \delta\left(E_{q}^{1}+E_{-q}^{1}-\varepsilon_{k}^{c}-\varepsilon_{k}^{v}\right) .
\end{aligned}
$$

Figure 1(b) diagrammatically depicts the matrix element $M$ of the Auger process. This diagram represents the interaction between the two initial $E_{11}$-excitons that results in the formation of a free $\mathrm{e}-\mathrm{h}$ pair as the final state. The population factors $\left(1-n_{k}^{c}\right)$ and $\left(1-n_{k}^{v}\right)$ for available scattering states of an electron and a hole are taken to be unity because the thermal populations of these states have negligible thermal populations. The AR rate is calculated to be $0.2 \mathrm{ps}^{-1}$ for a length of $1 \mu \mathrm{m} .^{20,26}$

Field dissociation of excitons, represented by $\Gamma_{\mathrm{F}}$ in Eqs. (1) and (2), is calculated using the Fowler-Nordheim equation, which is given by ${ }^{37}$

$$
\Gamma_{\mathrm{F}}(F)=\frac{E_{b}}{\hbar} \exp \left(-\frac{E_{b}^{3 / 2} m^{1 / 2}}{e F \hbar}\right),
$$

where $F$ is the dissociation field that is the same as the bias field in Eq. (3), $E_{b}$ is the exciton binding energy, and $m$ is the effective exciton mass.

Figure 2 shows photocurrent spectra for various field strengths. In this calculation, the laser intensity was fixed at $0.1 \mathrm{~W} / \mathrm{m}^{2}$. At an electric field strength of $1 \mathrm{~V} / \mu \mathrm{m}$, exciton states do not contribute to the photocurrents. The photocurrent spectrum has a one-dimensional asymmetric structure, which reflects the absorption spectrum of free electrons and holes in the one-dimensional structure. Under these conditions, carriers are generated from continuum states above the energy band gap.

In contrast, for electric field strengths of $6 \mathrm{~V} / \mu \mathrm{m}$ and higher, we find two new peaks associated with excitons at energies of about 0.7 and $1.5 \mathrm{eV}$. The lower peak is attributed to an $E_{11}$ exciton, while the higher asymmetric peak originates

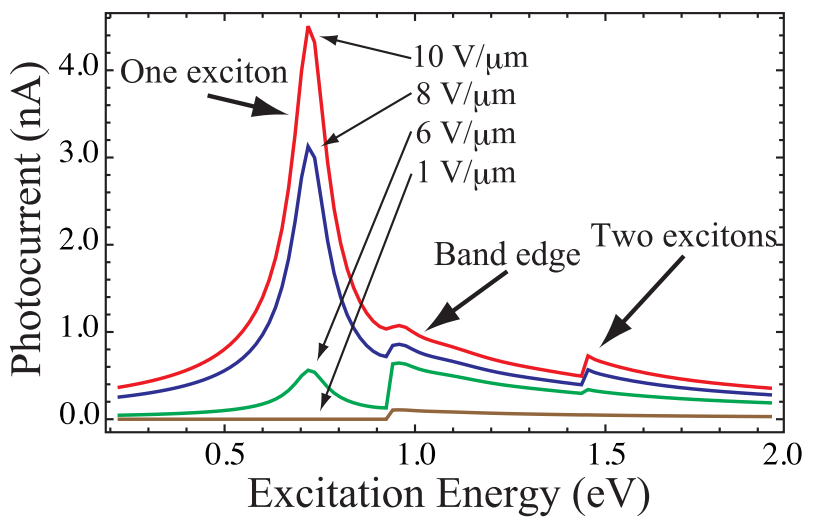

FIG. 2. Electric field dependence of photocurrents as a function of excitation energy. 


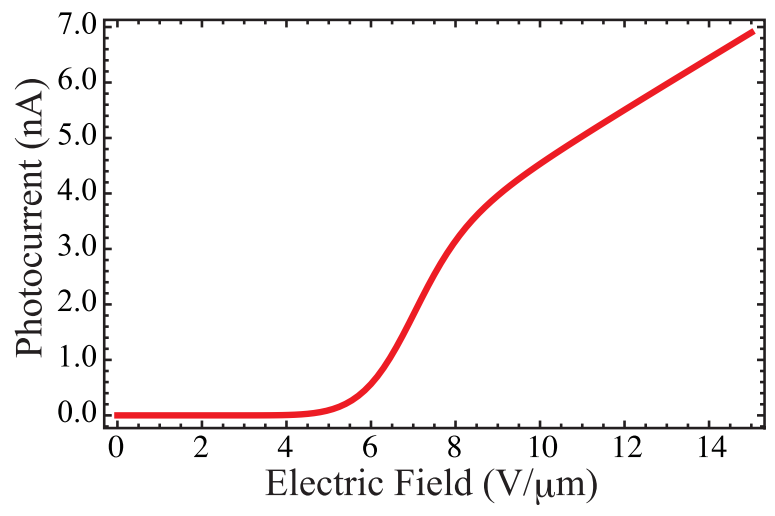

FIG. 3. Photocurrents as a function of electric field strength. Laser intensity is $0.1 \mathrm{~W} / \mathrm{m}^{2}$.

from the two-exciton state formed by MEG. The energy of the two-exciton state is twice that of the $E_{11}$ exciton. MEG increases the photocurrents relative to those from continuum states so that they exceed the threshold energy although the two excitons generated by MEG immediately recombine by AR. Thus, the total photocurrents are larger than those without MEG.

To analyze the electric field dependence of photocurrent spectra, Fig. 3 shows photocurrents at the exciton energy of $E_{11}$ exciton (i.e., the lowest bright exciton) as a function of the electric field. The exciton is found to dissociate at electric fields of $6 \mathrm{~V} / \mu \mathrm{m}$ or higher. Otherwise, excitons cannot contribute to photocurrents because they are electrically neutral.

Figure 4 shows the photocurrent generation efficiency of MEG excitons for various electric field strengths and a laser intensity of $0.1 \mathrm{~W} / \mathrm{m}^{2}$. At an electric field strength of $6.0 \mathrm{~V} / \mu \mathrm{m}$, the efficiency slightly exceeds $100 \%$. With increasing electric field strength, the efficiency increases, reaching the MEG efficiency. AR does not play any role due to the rapid dissociation by the electric field. This result directly demonstrates that MEG increases the total photocurrent.

Baer and Rabani proposed the impact excitation mechanism to explain the experimental result ${ }^{14}$ by solving the

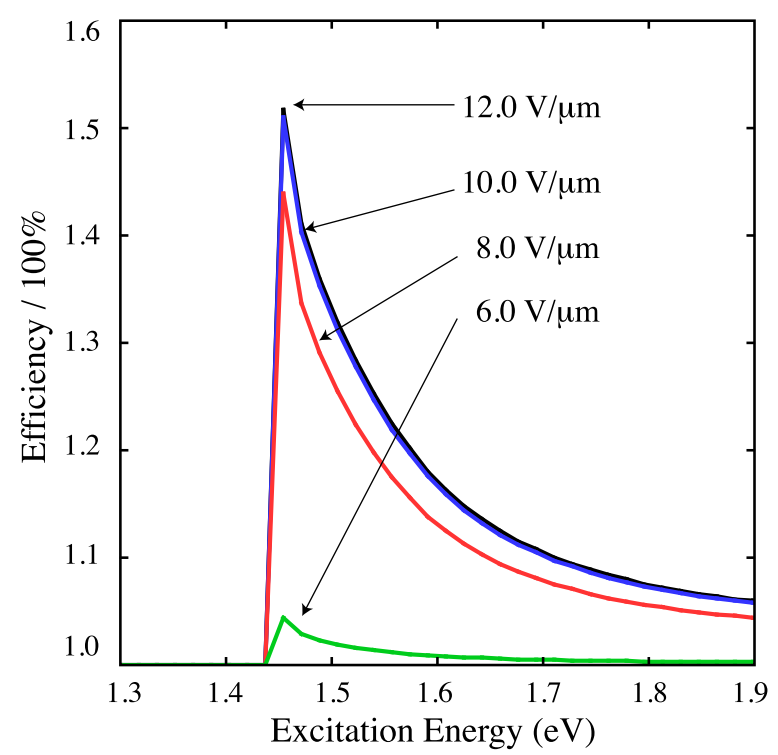

FIG. 4. Photocurrent generation efficiency for various electric field strengths. Laser intensity is fixed at $0.1 \mathrm{~W} / \mathrm{m}^{2}$.
Langevin equation including the energy dissipation due to the phonon scattering. ${ }^{18}$ In their mechanism, the initial quasi-bound excitonic state breaks into an electron in the third subband and a hole in first subband. Then those free carriers gain energies by the electric field, resulting in the efficient impact excitation.

On the other hand, our study focuses on many-body effects such as MEG and AR in photocurrent generation, but not on time-dependent problem. For the MEG mechanism, it is based on the direct generation of multiple excitons. ${ }^{19} \mathrm{We}$ think that both our and their results are crucial for photocurrent generation in SWCNTs and thus complement each other.

In summary, we have calculated the photocurrent in SWCNT by accounting for the effects of exciton interactions. Our calculation unravels the effects of the two competing processes, AR and MEG, on photocurrent generation and shows that these processes are crucial for determining the efficiency of photocurrent generation in SWCNTs. We found that MEG can compete with AR, which increases the photocurrent. SWCNTs are thus promising materials for highefficient photovoltaic devices.

This work was supported by the Japan Science and Technology Agency (CREST) and a Grant-in-Aid for Scientific Research from the Ministry of Education, Culture, Sports, Science and Technology (MEXT) of Japan.

${ }^{1}$ W. Shockley and H. J. Queisser, J. Appl. Phys. 32, 510 (1961).

${ }^{2}$ A. J. Nozik, Physica E 14, 115 (2002).

${ }^{3}$ R. D. Schaller and V. I. Klimov, Phys. Rev. Lett. 92, 186601 (2004).

${ }^{4}$ R. J. Ellingson, M. C. Beard, J. C. Johnson, P. Yu, O. I. Micic, A. J. Nozik, A. Shabaev, and A. L. Efros, Nano Lett. 5, 865 (2005).

${ }^{5}$ R. D. Schaller, J. M. Pietryga, and V. I. Klimov, Nano Lett. 7, 3469 (2007).

${ }^{6}$ M. C. Beard, K. P. Knutsen, P. Yu, J. M. Luther, Q. Song, W. K. Metzger, R. J. Ellingson, and A. J. Nozik, Nano Lett. 7, 2506 (2007).

${ }^{7}$ T. Ando, J. Phys. Soc. Jpn. 66, 1066 (1997).

${ }^{8}$ V. Perebeinos, J. Tersoff, and P. Avouris, Phys. Rev. Lett. 92, 257402 (2004).

${ }^{9}$ C. D. Spataru, S. Ismail-Beigi, L. X. Benedict, and S. G. Louie, Phys. Rev. Lett. 92, 077402 (2004).

${ }^{10}$ C. L. Kane and E. J. Mele, Phys. Rev. Lett. 90, 207401 (2003).

${ }^{11}$ H. Zhao and S. Mazumdar, Phys. Rev. Lett. 93, 157402 (2004).

${ }^{12}$ F. Wang, G. Dukovic, L. E. Brus, and T. F. Heinz, Science 308, 838 (2005).

${ }^{13}$ A. Ueda, K. Matsuda, T. Tayagaki, and Y. Kanemitsu, Appl. Phys. Lett. 92, 233105 (2008).

${ }^{14}$ N. M. Gabor, Z. Zhong, K. Bosnick, J. Park, and P. L. McEuen, Science 325, 1367 (2009).

${ }^{15}$ S. Wang, M. Khafizov, X. Tu, M. Zheng, and T. D. Krauss, Nano Lett. 10, 2381 (2010).

${ }^{16}$ Y. Li, S. Kodama, T. Kaneko, and R. Hatakeyama, Appl. Phys. Express 4, 065101 (2011).

${ }^{17}$ V. Perebeinos and P. Avouris, Phys. Rev. B 74, 121410(R) (2006).

${ }^{18}$ R. Baer and E. Rabani, Nano Lett. 10, 3277 (2010).

${ }^{19}$ S. Konabe and S. Okada, Phys. Rev. Lett. 108, 227401 (2012).

${ }^{20}$ F. Wang, Y. Wu, M. S. Hybertsen, and T. F. Heinz, Phys. Rev. B 73, 245424 (2006).

${ }^{21}$ K. Matsuda, T. Inoue, Y. Murakami, S. Maruyama, and Y. Kanemitsu, Phys. Rev. B 77, 033406 (2008).

${ }^{22}$ Y. Murakami and J. Kono, Phys. Rev. Lett. 102, 037401 (2009).

${ }^{23}$ Y. Murakami and J. Kono, Phys. Rev. B 80, 035432 (2009).

${ }^{24}$ Y. F. Xiao, T. Q. Nhan, M. W. B. Wilson, and J. M. Fraser, Phys. Rev. Lett. 104, 017401 (2010).

${ }^{25}$ S. Moritsubo, T. Murai, T. Shimada, Y. Murakami, S. Chiashi, S. Maruyama, and Y. K. Kato, Phys. Rev. Lett. 104, 247402 (2010). 
${ }^{26} \mathrm{~S}$. Konabe, T. Yamamoto, and K. Watanabe, Appl. Phys. Express 2, 092202 (2009).

${ }^{27}$ S. Konabe and S. Okada, New J. Phys. 14, 023053 (2012).

${ }^{28}$ D. A. Stewart and F. Léonard, Phys. Rev. Lett. 93, 107401 (2004).

${ }^{29}$ J. Guo, M. A. Alam, and Y. Yoon, Appl. Phys. Lett. 88, 133111 (2006).

${ }^{30}$ D. Hirai and S. Konabe, Appl. Phys. Lett. 99, 223103 (2011).

${ }^{31}$ V. Perebeinos, J. Tersoff, and P. Avouris, Phys. Rev. Lett. 94, 027402 (2005).

${ }^{32}$ V. Perebeinos, J. Tersoff, and P. Avouris, Nano Lett. 5, 2495 (2005).
${ }^{33}$ V. Perebeinos, J. Tersoff, and P. Avouris, Phys. Rev. Lett. 94, 086802 (2005).

${ }^{34}$ T. Ando, J. Phys. Soc. Jpn. 75, 024707 (2006).

${ }^{35}$ J. Jiang, R. Saito, Ge. G. Samsonidze, A. Jorio, S. G. Chou, G. Dresselhaus, and M. S. Dresselhaus, Phys. Rev. B 75, 035407 (2007).

${ }^{36}$ R. B. Capaz, C. D. Spataru, S. Ismail-Beigi, and S. G. Louie, Phys. Rev. B 74, 121401 (2006).

${ }^{37}$ V. Perebeinos and P. Avouris, Nano Lett. 7, 609 (2007). 